TTR

Traduction, terminologie, re?daction

\title{
Herméneutique et traduction poétique : quelques remarques
}

\section{Érik Vigneault}

Volume 12, numéro 2, 2e semestre 1999

Poésie, cognition, traduction II - Autour d'un poème de W. H. Auden

Poetry, Cognition, Translation II — On a Poem by W. H. Auden

URI : https://id.erudit.org/iderudit/037378ar

DOI : https://doi.org/10.7202/037378ar

Aller au sommaire du numéro

\section{Éditeur(s)}

Association canadienne de traductologie

\section{ISSN}

0835-8443 (imprimé)

1708-2188 (numérique)

Découvrir la revue

\section{Citer cet article}

Vigneault, É. (1999). Herméneutique et traduction poétique : quelques remarques. TTR, 12(2), 173-188. https://doi.org/10.7202/037378ar

\section{Résumé de l'article}

Herméneutique et traduction poétique : quelques remarques - Une approche herméneutique de la traduction ne doit pas se résumer au simple énoncé selon lequel il faut avoir compris un texte pour le traduire. Elle doit viser à dégager ce que je nomme dans cet article l'intention du texte. Je tente dans ces pages de cerner cette notion d'intention en recourant tout d'abord à une comparaison de la traduction philosophique et poétique, ensuite à l'analyse de deux poèmes : l'un de Ludwig Greve, l'autre de Thomas Bernhard, dont je critique la traduction donnée par Jean-Pierre Lefebvre et duquel je propose une nouvelle version française.
Tous droits réservés (c) TTR: traduction, terminologie, rédaction — Les auteurs, 1999
Ce document est protégé par la loi sur le droit d'auteur. L’utilisation des services d'Érudit (y compris la reproduction) est assujettie à sa politique d'utilisation que vous pouvez consulter en ligne.

https://apropos.erudit.org/fr/usagers/politique-dutilisation/ 


\section{Herméneutique et traduction poétique : quelques remarques}

\section{Érik Vigneault}

L'herméneutique de la traduction ne doit pas se laisser restreindre au banal énoncé selon lequel "il faut avoir compris un texte pour le traduire ". À moins de faire glisser cette affirmation sur le terrain de la philosophie et de reconnaître que la notion de compréhension est en soi une question fort problématique, le caractère trop évident de cette phrase lui dérobe toute portée et la réduit à un simple truisme. L'herméneutique opère à un autre niveau que celui trop simpliste de la compréhension de l'objet à traduire.

Une certaine critique l'accuse de n'être réceptive qu'au sens, de ne pas avoir de théorie du texte, de ne pas se préoccuper de ce que le texte fait. Je crois qu'elle a en partie raison, mais en partie seulement car cette conception repose sur un malentendu. L'examen de traductions de poésies révèle que de nombreux traducteurs croient pouvoir isoler la teneur sémantique d'un poème de sa forme, teneur qu'ils s'attachent ensuite à rendre dans la langue d'arrivée. La maîtrise de ce qu'ils considèrent être "le sens" serait le gage d'une bonne traduction. Cette manière d'aborder la traduction relève toutefois non pas tant d'une conception herméneutique du traduire que d'un manque de sensibilité à ce qu'est un poème et d'une certaine naïveté. D'abord, et il s'agit là d'un principe de l'herméneutique, parce qu'il n'y a pas dans un poème un sens objectif qu'un lecteur attentif arriverait à dégager; la compréhension d'un poème (mais aussi de tout texte, de tout énoncé) est un processus complexe qui engage avec lui les préjugés (Vorurteile chez Gadamer) de l'interprète (aspect trop souvent négligé dans l'herméneutique littéraire), donc une grande part de subjectivité dont il faut être conscient. Ensuite, parce que la manière 
d'être d'un poème est constitutive de sa signification : le signifiant et le signifié sont étroitement imbriqués et forment un dense tissu; considérer l'un sans l'autre conduit nécessairement à manquer l'un et l'autre. C'est ainsi que la prétendue herméneutique de ces traducteurs se résume le plus souvent à une sémantique du mot qui rate la valeur intrinsèque du poème.

Ces quelques remarques visent à esquisser une approche herméneutique de la traduction qui tienne compte du tout du poème. La notion clef me paraît être ici celle de l'intention du texte - une manière de vouloir-dire qui résulte de la somme des éléments signifiants du texte -, qui ne doit pas être confondue avec l'intention de l'auteur; souvent prise pour l'objet de l'herméneutique. Ces remarques sont réparties en quatre sections. J'entends d'abord montrer, en prenant comme exemple un poème de Ludwig Greve (1924-1991), que l'herméneutique peut et doit s'aider d'un examen formel du poème si elle veut proposer une approche satisfaisante de la traduction. Bien que j'aie en vue principalement la poésie, je me permettrai dans un deuxième temps un bref détour par la traduction de philosophie, incursion qui m'aidera à définir le concept d'intention. Je proposerai finalement une lecture ainsi qu'une nouvelle traduction d'un poème de Thomas Bernhard déjà mis en français par Jean-Pierre Lefebvre.

L'herméneutique doit aborder un poème dans sa signifiance globale, tissée par l'entrelacement de tous ses éléments. Il lui faut viser la manière d'être et de faire du texte si elle veut toucher à l'essentiel, et non se limiter à une interprétation ponctuelle. Car considérer un mot isolément de l'ensemble conduit nécessairement à une interprétation fragmentaire : les mots d'un poème deviennent ce que le poème en fait. Cette remarque n'est pas sans importance pour la traduction. La poésie traduite a souvent un air de mot-à-mot esthétisé. J'aimerais montrer par un poème de Ludwig Greve $(1992$, p. 7) comment la lecture combinée du vouloir-dire d'un poème et de sa manière d'être peuvent en guider l'interprétation et par suite aiguiller les choix du traducteur. Voici d'abord le poème en entier : 


\section{Mein Vater}

1 Spät komme ich zu dir.

2 Wenn Staub mich riefe - aber ich höre nur

im Spiel der feuchten, meiner Lippen, diese gehorsame Stimme rufen.

5 Wo niemand wartet, Vater, im Schweigen, wo

6 in Salz und Asche kenne ich deinen Mund, der nach den Kindern ruft und ächzend

bittet um Gnade die Menschensöhne.

9 Die ehmals gute Jacke verriet den Herrn.

10 Du ohne Mantel, war auch kein Tier dabei noch Gott : wie sorgsam fuhrtest du in zwiefacher Kälte dein Kind zur Grube.

13 Dein Aug, die Stime, Tafel vom Sinai, 14 der Nase starker Bogen - ich sehe nichts und halte Nase, Stirn und deine Bitternis doch in den hohlen Händen.

17 Ja, diese Hand, die unschlüssig Wort auf Wort

18 hier fügt, sie ahmte lange die Bögen nach von deinem Namen, übte heimlich Strenge und Mut des gerechten Mannes,

21 dem ich nie sagen konnte : ich bin dein Sohn.

22 Man hieß uns Fremde. Unsere Sprache war ein Blick, ein Handetausch, und später Auflehnung, bleiche Gewalt des Zornes.

25 Genügt die Trauer ? Atem, Begeisterung,

26 die Liebesnächte danke ich deinem Grab

27

28 und auch die Kinder : unerschöpflich höre sie lachen... Ich komme, Vater.

Ludwig Greve a construit son poème sur une forme fixe de la métrique allemande, depuis longtemps à peu près abandonnée, l'ode alcaïque, dont le schéma est le suivant ( $u=$ syllabe non accentuée; - = syllabe accentuée) : 


$$
\begin{aligned}
& u-u-u-u u-u- \\
& u-u-u-u u-u- \\
& u-u-u-u-u \\
& -u-u-u u-u-u
\end{aligned}
$$

Le verbe hören (ÉCOUTER, ENTENDRE) du tout dernier vers (28) du poème (unerschöpflich / höre sie lachen... Ich komme, Vater) pose un problème d'interprétation et par conséquent de traduction. En raison de l'absence d'un sujet immédiatement visible, il y a en effet au moins deux manières de le lire : comme indicatif présent conjugué à la première personne du singulier : J'ÉCOUTE (J'ENTENDS), ou comme impératif à la deuxième personne : ÉCOUTE (ENTENDS) ${ }^{1}$. La nuance est d'importance. Dans le premier cas, c'est le je du poème qui écoute, celui qui tout au long du texte s'adresse au père disparu. Dans le deuxième, c'est le père qui entend, qui aurait la possibilité d'entendre, c'est dire qu'il aurait toujours une certaine présence, une forme de proximité, de vie, et c'est tout le poème qui devrait être relu à la lumière de cette présence inattendue révélée au dernier vers. L'ambiguîté, dont l'auteur est certainement conscient, ouvre au poème un nouvel horizon, et si cela était possible, le traducteur devrait s'efforcer de la conserver. Or il se trouve que le français n'arrive pas à maintenir les deux possibilités. Le traducteur se voit dans la désagréable position de devoir opter pour un sens au détriment de l'autre.

L'aspect de la phrase, caractérisée par le deux-points qui la précède, invite à considérer la phrase comme complète et donc à lire le verbe comme impératif. D'un point de vue syntaxique, c'est la lecture qui semble naturelle. Pourtant, la tension sémantique du poème (ce $q u$ 'il faudrait démontrer par une lecture en profondeur du poème, pour laquelle je n'ai pas de place ici) nous amène plutôt à voir dans le sujet de ce verbe non pas le père mais le je. Höre serait donc un indicatif présent, dont le sujet, $i c h$, se trouverait deux vers plus haut, déjà sujet de danke.

Mais il faut alors s'interroger sur l'omission du sujet dans cette phrase qui semble complète sans celui-ci et se questionner sur la

\footnotetext{
${ }^{1}$ Il pourrait également être à la première personne du subjonctif présent, mais cette possibilité, toute admissible qu'elle soit sur la plan grammatical, ne se défend pas dans le contexte.
} 
signification de cette absence, vérifier si elle peut s'expliquer. Je rejette l'explication selon laquelle le poète aurait simplement cherché à éviter la répétition : le pronom ich apparaît déjà huit fois dans le poème, dont deux fois dans le vers 21 . À la justification qui nous est donnée par une interprétation du poème peut s'en greffer une autre que l'on obtient par l'analyse de la métrique. J'ai reproduit ci-dessus le schéma de l'ode alcaïque, que le poème épouse rigoureusement (sauf pour ce qui est du $1^{\text {er }}$ vers, qui ne compte que trois unités iambiques). Or l'inscription d'un ich comme sujet du verbe höre aurait comme conséquence de briser la séquence rythmique et ajouterait une onzième syllabe à un vers qui ne doit en contenir que dix. De plus, au $9^{\mathfrak{c}}$ vers (Die ehmals gute Jacke verriet den Herrn), Greve n'a pas hésité à recourir à une orthographe archaïque du mot ehemals pour respecter le schéma de son ode : il en supprime le $2^{\mathrm{e}}$ e (ehmals au lieu de ehemals), obtenant ainsi deux syllabes : $-u$, et non trois : $-u-$. Aucun dictionnaire moderne ne fait plus mention de cette orthographe (que l'on retrouve encore chez Hölderlin, par exemple). Même chose au vers 13, où le poète a omis le $e$ final du mot $A$ uge (procédé très courant à l'époque où l'ode était une forme répandue - chez Hölderlin bien sûr, mais aussi Klopstock, l'un des maîtres de Greve). On constate que Greve n'hésite pas à s'écarter de l'orthographe admise pour travailler dans la forme qu'il s'est donnée. Il est donc permis de croire que l'absence du sujet du verbe höre soit elle aussi le résultat d'un écart syntaxique qui serait motivé par des raisons métriques, et d'en conclure, à la lumière d'une analyse tant sémantique que formelle, que le verbe höre est un indicatif présent à la $1^{\text {re }}$ personne du singulier, dont le sujet est le pronom ich du vers 26 .

Certes, dans le texte allemand les deux possibilités demeurent inscrites, et il n'est pas interdit de lire le poème doublement. Mais le français n'arrivant pas à conserver cette tension entre les deux possibilités, pour les raisons évoquées plus haut je traduirais : JE LES ENTENDS RIRE (et non ÉCOUTE-LES RIRE, Ou ENTENDS-LES RIRE). Le lecteur de la traduction ne se doutera pas de la tension sémantique que comporte l'origınal. Peu importe l'option qu'il privilégiera, le traducteur se trouvera à interpréter. Sa tâche ultime est de décider en faveur du texte, c'est-à-dire dans le sens de son intention, qui ne peut être mise au jour que par une lecture globale des éléments signifiants du poème. Cette lecture globale constitue l'approche herméneutique de la traduction. En dernière analyse, c'est le texte qui dicte comment traduire. 
On ne lit ni ne traduit de la même manière un traité philosophique et un poème. C'est en tout cas la conclusion empirique à laquelle il faut en venir après s'être livré à l'examen comparatif de traductions philosophiques et poétiques. Dans la traduction française de la Critique de la raison pure de Jules Barni, revue par P. Archambault, une « Note sur le texte de cette édition" précise : "Nous avons mis entre crochets tous les mots ou membres de phrases qui, ajoutés par J. Barni et nécessaires à l'intelligence du texte français, n'ont pas d'équivalents dans le texte allemand " (Kant, 1987, p. 24; c'est moi qui souligne). Un rapide coup d'œil au texte nous permet de constater le nombre important de ces retouches. Dans la même veine, les traducteurs de la Troisième Critique de Kant (1985), publiée sous la direction de Ferdinand Alquié, n'ont pas hésité à " compléter » le texte, dont le style est souvent fort confus, pour le rendre plus clair. Par exemple, là où le philosophe se contente d'un pronom pour remplacer un nom lointain, ou lorsque ce pronom peut logiquement remplacer deux noms, la traduction donne un texte plus explicite en répétant le nom; là où le texte est trop allusif, les traducteurs ont ajouté une phrase pour que l'on suive bien la pensée de Kant. Plusieurs passages pourraient être cités. Il s'agit presque toujours de furtives interventions qui n'ont pas comme objectif d'amender le texte mais celui de le rendre plus accessible. Voici un demier exemple : dans Wahrheit und Methode (1990, p. 296), Gadamer utilise l'expression sprachliche Bedeutung, que les traducteurs ont rendue par « signification d'ensemble, telle qu'elle est présente dans son expression linguistique " (trad. franç., p. 312). Les traducteurs n'ont pas voulu sacrifier la précision à la concision. Car si la tournure ne brille pas par son élégance, elle a du moins le mérite d'être claire. Les traducteurs ont interprété le sens de l'adjectif sprachlich et l'ont explicité dans leur adaptation. Trois exemples, parmi tant d'autres, où les traducteurs n'hésitent pas à proposer une traduction plus explicite que l'original. Ceci me rappelle une amusante anecdote que Jacques Brault relate dans Chemin faisant, qui n'est pas sans intérêt pour notre propos. Faisant part à un ami allemand, philosophe de profession, de son désir d'apprendre sa langue dans le but de lire Kant, Hegel et d'autres dans le texte, J. Brault s'entendit répondre (je cite de mémoire) : ne vous donnez pas cette peine; quand je veux comprendre Hegel, je le lis en français. Boutade qui fait sourire, mais qui n'invite pas moins à la réflexion. Nous avons vu que les traducteurs pouvaient être guidés par un souci de clarté et de précision, qui peuvent 
éventuellement faire défaut à l'original. Un travail d'explicitation se fait alors à l'intérieur même du processus de traduction. Cette manière d'expliciter est ce qui fait dire à Gadamer que « Toute traduction qui prend au sérieux sa tâche est plus claire mais aussi plus plate que l'original ${ }^{2}$ » (Gadamer, trad. franç., p. 408; c'est moi qui souligne). On peut tirer de ce qui précède la conclusion suivante, qu'une traduction peut renfermer une part d'interprétation ${ }^{3}$. Le traducteur s'autorise de son interprétation pour expliciter. Il comprend ce qui est en jeu, ce que le texte veut dire, et il tente de rendre ce vouloir-dire aussi clairement que possible en rectifiant les éventuelles maladresses de l'original qui rendent sa lecture malaisée.

Il ne fait pas de doute cependant que le caractère explicite de ce type de traduction convienne mal à la poésie, que traduire Rilke comme on traduirait Kant relèverait d'un manque de jugement criant. C'est là le sens de la critique que formule Philippe Jaccottet à l'endroit

${ }^{2}$ " Jede Übersetzung, die ihre Aufgabe ernst nimmt, ist klarer und flacher als das Original » (Gadamer, 1990, p. 390).

${ }^{3}$ On peut même radicaliser cet énoncé et affirmer que toute traduction est interprétation Cette thèse se défend diversement, notamment par le fait que pratiquement tous les mots d'une langue peuvent avoir de multiples acceptions, dont habituellement une seule est vivante et visée dans un contexte donné. Le plus souvent, le contexte nous permet de discerner le sens, sans qu'il soit besoin de se livrer à un complexe exercice d'interprétation; d'autres fois cependant le sens ne se laisse pas si facilement saisir et il faut procéder à une interprétation plus systématique. Mais dans tous les cas nous interprétons, et le traducteur également. Je me contente d'un bref exemple. On devine assez facilement l'embarras du lecteur allemand peu familier avec la pensée de Hegel, qui, ouvrant Die Phenomenologie des Geistes voit surgir devant lui ce mot de aufheben. Le recours au dictionnaire ne lui sera pas d'un grand secours : s'agit-il de lever, de relever, ou bien de garder, de conserver, de mettre de côté, ou encore, presque à l'inverse, d'abolir, d'abroger, d'annuler? Le lecteur devra jouer les interprètes, se torturer les méninges pour déterminer lequel de ces sens attribuer à l'expression. Au pire, il pourra poursuivre sa lecture avec une idée un peu vague. Mais le traducteur, lui, n'a pas cette liberte. Il doit trancher, et en tranchant il se trouve à interpréter pour son lecteur. Par ce fait même qu'elle est une prise de position, une interprétation, une affirmation d'un sens au détriment d'un autre, la traduction peut devenir une manière de commentaire de l'original, commentaire qui ne prend pas la forme d'une glose accompagnant le texte dans ses passages les plus obscurs, mais qui s'actualise plutôt implicitement dans les choix que le traducteur fait. 
d'une traduction que donnait Armel Guerne des Élégies de Duino. Jaccottet écrit de cette traduction que :

Elle cherche d'un bout à l'autre à rendre fidèlement, intégralement, le sens de l'œuvre dans ses moindres nuances; elle témoigne que celuici a été compris en profondeur [...] Mais elle n'est pas cette ouvre essentiellement musicale, cette réussite verbale (impossible peutêtre).

Jaccottet analyse une suite d'images, qui s'ouvre sur les mots : Frühe Geglückte,

[...] dont chacun comporte au moins deux nuances; früh signifiant à la fois "premier" et "précoce, matinal ", évoquant à la fois le commencement et le matin; geglückt voulant dire réussi, mais avec la nuance de bonheur que nous donne en français l'expression "une heureuse formule ». Armel Guerne traduit : "Réussites heureuses et tôt parfaites », prouvant ainsi qu'aucune nuance de l'expression ne lui a échappé, et qu'une grande honnêteté l'a contraint à les rendre toutes; mais qui ne voit qu'il a dû sacrifier ainsi à la fois la concision, l'audace, la fermeté et la sonorité flûtée de l'original? (Jaccottet, 1987, pp. 136-137)

Certes Guerne a compris le texte. Mais il s'en tient au message du poème, il en néglige sa manière d'être, et ce faisant il en néglige un aspect constitutif : cette concision, cette fermeté, cette sonorité dont parle Jaccottet et qui font que le poème de Rilke est un grand poème et non simplement un ensemble de mots porteurs d'un message. Sa traduction pêche précisément par cela qu'on louerait dans la traduction d'un texte philosophique : une fidèle explicitation du sens. Le souci de rendre les nuances sémantiques de l'allemand s'est avéré pour Guerne plus pressant que celui de faire un poème.

Le parti pris d'explicitation que nous avons observé dans les adaptations philosophiques ne répond pas aux exigences d'une traduction de poésie. Il y aurait donc une différence entre traduire de la poésie et traduire de la philosophie. Il me semble que la différence ne relève pas du genre, mais bien de l'intention du texte, et que c'est sur la base de cette intention que l'on est amené à traduire différemment un poème et un traité de philosophie. 
L'intention du texte est la dynamique qui résulte de l'ensemble des éléments qui ont valeur dans un texte donné : valeur sémantique, rythmique, phonétique, etc., tout ce qui signifie et qui fait que le texte est ce qu'il est - une sorte de vouloir-dire du texte par sa manière d'être. Traduire demande la mise au jour de cette intention et sa recréation. Il ne saurait être question d'une sémantique du mot : le mot hors de son texte est abstrait, ce n'est que dans la cohabitation avec tous les autres mots qu'il prend corps, qu'il devient vivant, qu'il acquiert sa valeur. La valeur d'un signe dans un poème déborde le signe en soi pour devenir ce que le poème fait de lui. On peut en ce sens parler de cercle herméneutique, car chacun des éléments est apprécié dans le contexte global que constitue le texte, qui lui-même existe par le tout de ses éléments.

Qu'il s'agisse d'un traité philosophique, d'un poème ou d'un roman, la tâche demeure toujours la même. Seuls les moyens appropriés pour recréer une même intention diffèrent, parce que les moyens mis en œuvre pour créer l'original sont propres à chaque texte. Il n'y a pas deux approches du traduire : il y a plusieurs manières de traduire qui relèvent toutes d'une même approche.

Puisque c'est l'intention du texte qui est déterminante, la question de savoir s'il faut par principe traduire en vers un poème versifié est en soi une fausse question (qui nous renvoie à la vieille croyance selon laquelle ce qui est écrit en vers est de la poésie, le reste de la prose). Car il n'y a pas un oui ou un non qui vaille absolument. Dans chaque cas la question doit être reposée, la réponse jugée à l'aune de l'intention de l'original. La pratique de la traduction nous plonge nécessairement dans un délicat jeu de compromis qui nous oblige à privilégier tel aspect au détriment de tel autre. Une bonne traduction sera celle qui saura recréer un texte à partir des éléments clés du texte de départ - ce qui ne peut s'accomplir que si l'on a préalablement su mettre au jour l'intention du texte. La tâche du traducteur est donc de saisir tout ce qui signifie dans le texte, de sentir ses tensions profondes, son intention - et de là de recréer un texte mu par la même intention, avec des moyens propres à la langue d'arrivée. Qui traduit en vers un poème en vers, qui met des rimes là où il $\mathrm{y}$ a des rimes n'a rien fait s'il n'a pas rendu l'intention du poème. Car ce n'est pas la forme choisie 
qui est déterminante, c'est l'intention. Il n'y a pas en soi de forme équivalente; l'équivalence se situe à un niveau plus fondamental.

L'intention du texte me semble être précisément ce à quoi Jean-Pierre Lefebvre n'a pas été sensible dans la traduction qu'il donne du poème "Warum fürchte ich mein Altern... " (Lefebvre, 1995, pp. 1326-1327) de Thomas Bernhard. Voici le poème et l'adaptation de J.P. Lefebvre :

\section{Poème original de Thomas Bernhard}

1 Warum furrchte ich mein Altern

2 meinen Tod der mich befällt

3 den Schrei ?

4 Ich fürchte mich o Herr

5 ich furchte meine Seele

6 und den Tag der and der Mauer lehnt

7 und mich zersägt

8 o Herr

9 ich fürchte mich

10 ich fürchte schon die Nacht

11 die vor den Dörfern steht

12 und hinterm Haus

13 die in den Kühen heult

14 und mit den Sternen tanzt

15 o Gott

16 ich fürchte mich

17 vor Dir

18 und vor der Traurigkeit ${ }^{4}$

19 die mir den Mund zerschlägt

20 ich furchte Herr

21 mein Grab

22 und mein Geschick in Düsternis

23 o Herr den Tod

\section{Traduction de Jean-Pierre Lefebvre}

Pourquoi ai-je peur de vieillir de ma mort qui me frappera, du cri?

J'ai peur, ô Seigneur j'ai peur de mon âme et du jour appuyé sur le mur et qui me cisaille ô Seigneur j'ai peur j'ai déjà peur de la nuit qui est au bord des villages et derrière la maison qui hurle dans les vaches et danse avec les astres ô Dieu j'ai peur de Toi et de ta tristesse qui m'écrase la bouche j'ai peur Seigneur de ma tombe et de mon sort dans la sinistre ténèbre Ô Seigneur de la mort.

4 Une coquille s'est glissée dans la Pléiade : Traurigkeit est écrit Traurigheit. J'ai rectifié. 
Le poème est constitué de deux phrases comportant une ponctuation finale : la première est à la forme interrogative et elle comprend les 3 premiers vers; la seconde, affirmative, compte les 20 vers suivants. Ni l'une ni l'autre ne présentent de ponctuation interne. On remarquera également que le poème ne contient aucun adjectif et que tous les verbes sont conjugués au présent de l'indicatif. Le texte est construit autour de l'expression ich fürchte mich, qui acquiert une valeur toute particulière dans ce poème. Bernhard exploite ses possibilités et l'utilise de trois manières : avec $1^{\circ}$ un complément à l'accusatif qui est l'objet de la peur (ex. : vers 5 : ich fürchte meine Seele - J'AI PEUR DE MON ÂME), $2^{\circ}$ un pronom réfléchi et un complément au génitif introduit par la préposition vor (ex. : vers 16-17 : ich fürchte mich / vor Dir), et $3^{\circ}$ un pronom réfléchi à l'accusatif (ex.: vers $4:$ ich fürchte mich). Ce dernier emploi peut être compris de deux façons dans ce poème. Dans le premier cas, il s'agit de l'emploi ordinaire de l'expression qui signifie J'AI PEUR. Mais le poème nous invite à la lire d'une deuxième manière : en considérant le pronom mich non pas comme pronom réfléchi mais comme objet à l'accusatif (la première des trois utilisations présentées ci-dessus). Cette façon inusitée de comprendre ich fürchte mich est donnée par la manière même du poème de juxtaposer des compléments à l'accusatif. Ainsi, chacun des trois premiers vers comporte un accusatif : ich fürchte mein Altern (vers 1), meinen Tod (vers 2) et den Schrei (vers 3); le lecteur est ainsi porté, presque naturellement, à voir dans le mich du $4^{\mathrm{e}}$ vers un complément direct au même titre que mein Altern, meinen Tod, etc. Ich fürchte mich dirait tout à la fois : J'AI PEUR et J'AI PEUR DE MOI. Sur ce jeu repose l'une des marques du texte.

La possibilité sémantique que le pronom mich soit objet de la peur donne un tout autre éclairage au poème. Le je est effrayé par tout, depuis son vieillissement jusqu'à sa mort, depuis son âme jusqu'à Dieu. Qu'il puisse être effrayé par lui-même le montre dans un état de frayeur totale : l'objet de la peur se trouve partout autour de lui et en lui-même, le je ne peut y échapper. Celui qui aurait peur de tout sauf de soi pourrait se réfugier en lui-même; or cette possibilité est refusée au je par le fait qu'il est lui-même le propre objet de sa peur.

Jean-Pierre Lefebvre rend ich fürchte mich par J'AI PEUR, qui en est la traduction naturelle. Or ce choix me semble ne pas tenir compte de la valeur de l'expression allemande dans le poème. Pour les raisons évoquées, je proposerais plutôt JE M'EFFRAIE. Cette tournure 
inusitée présente des avantages phonétiques. Dans l'allemand ich fürchte mich, l'allitération des trois $c h$ s'entend très nettement; en outre, le ich se retrouve dans le mich et forme avec lui une boucle: l'objet contient le sujet. En français, le pronom réfléchi forme avec le pronom possessif de plusieurs compléments une allitération: JE M'EFFRAIE DE MON DÉCLIN (vers 1), DE MA MORT (double allitération, vers 2 ), de MON CRJ (vers 3 ), etc. Mais surtout, le pronom réfléchi, à la manière du mich dans l'expression allemande, peut devenir, symboliquement du moins, l'objet de la frayeur: je m'effraie de moimême, j'effraie moi-même, je m'effraie. Eu égard à ma lecture du poème, à la valeur que la tournure allemande me paraît avoir, eu égard en somme à l'intention du poème, J'AI PEUR est une solution un peu frileuse; tout le poème demande une expression plus forte, plus riche, plus complexe.

En plusieurs autres points la traduction de Jean-Pierre Lefebvre me semble en deçà de l'original. En voici les principaux :

Vers 2 - Virgule après le verbe FRAPPERA, qui donne l'impression que le groupe verbal J'AI PEUR a deux objets, savoir 1) DE VIELLIR, qui aurait comme complément tout le vers 2 , et 2) DU CRI, tandis que Bernhard a donné trois objets à ich fürchte. L'allemand indique clairement que DE MA MORT du vers 2 n'est pas complément de VIEILLIR (cela donnerait " vieillir de ma mort ») mais de J'AI PEUR; les déclinaisons allemandes suffisent pour bien marquer les compléments, de sorte qu'aucune confusion de lecture n'est possible. La présence d'un verbe pour traduire un substantif (VIELLIR est censé rendre mein Altern) et d'une virgule fait naître dans la version de J.-P. Lefebvre une ambiguitté sémantique qui n'existe pas en allemand et qui rompt le rythme. Pour lever la confusion et redonner au poème sa cadence, je restitue les trois substantifs (DÉCLIN, MORT et CRI) et supprime la virgule. Par ailleurs, le verbe du vers 2 , befällt, est au présent, ce qui confère à la mort une présence particulière, malheureusement reléguée par le futur du français à une hypothétique et lointaine éventualité. Le travail de la mort est déjà commencé dans le poème de Bernhard, il est différé dans la traduction. En outre, le $t$ final de befällt fait écho aux deux $t$ de $\operatorname{Tod}$ (le $d$ se prononce ici comme un $t$ ). En proposant RONGE (au lieu de FRAPPERAI) je conserve le présent et tente de faire parler une autre allitération (déja existante chez J.-P. L.) : le $r$ de RONGE, déjà présent dans JE M'EFFRAIE, rappelle celui de MORT. 
$\mathrm{Au}$ vers 4 , une autre virgule a été rajoutée au français, qui elle aussi brise le rythme voulu par le texte. Je l'ai supprimée.

Dans les vers 11 et 12 , le texte se présente dans une opposition entre vor et hinter, opposition qui disparaît en français avec l'utilisation d'AU BORD et de DERRIÈRE. Je propose DEVANT et DERRIĖRE.

Au vers 7, la présence du und en allemand permet de faire le lien entre les verbes conjugués lehnt et zersägt, et de bien montrer que le pronom der (qui remplace Tag) est le sujet de chacun. Jean-Pierre Lefebvre a omis le ET, si bien que l'on prend MUR et non JOUR pour sujet de CISAILLE. L'ajout en français d'un ET rectifie la lecture.

Les verbes conjugués zersägt et zerschlägt (respectivement vers 7 et 19) se font écho; dans le version de Jean-Pierre Lefebvre, tout lien est rompu par l'utilisation de CISAILLE et ÉCRASE - je propose le couple CISAILLE/ASSAILLE, avec une inversion dans le dernier cas qui met en évidence le verbe et ainsi souligne le lien.

Au vers 22 , le traducteur a cru bon d'utiliser un adjectif. Or le poème allemand n'en contient aucun, ce qui est en soi l'une de ses caractéristiques. Par cet ajout le français ruine cette particularité, ajout que ne commande du reste aucune contrainte (qu'elle soit d'ordre sémantique ou formelle). La tentation de faire jouer l'allitération entre SORT et SINISTRE l'aura probablement emporté sur le désir de travailler dans l'intention du poème. Il s'agit d'un procédé de poétisation, motivée par le goût d'une certaine élégance qui n'est pas opportune.

Jean-Pierre Lefebvre a coupé le déroulement du poème en mettant fin à sa deuxième et dernière phrase à la fin de ce vers 22 , alors que l'allemand fait coïncider la fin de la phrase avec celle du poème. La traduction isole de ce fait le demier vers (23), et incite à voir dans le mot MORT un génitif de SEIGNEUR, au lieu que l'article à l'accusatif de l'allemand relie DE LA MORT (vers 23) à J'AI PEUR (vers 20).

L'ensemble de ces remarques m'amène à proposer la traduction suivante du poème de Thomas Bernhard :

1 Pourquoi m'effrayé-je de mon déclin

2 de ma mort qui me ronge

3 du cri? 
4 Je m'effraie ô Seigneur

5 je m'effraie de mon âme

6 et du jour appuyé au mur

7 et qui me cisaille

8 ô Seigneur

9 je m'effraie

10 je m'effraie déjà de la nuit

11 qui se tient devant les villages

12 et derrière la maison

13 qui hurle dans les vaches

14 et danse avec les astres

15 ô Dieu

16 je m'effraie

17 de Toi

18 et de la tristesse

19 qui ma bouche assaille

20 je m'effraie Seigneur

21 de ma tombe

22 et de mon sort dans la ténèbre

23 ô Seigneur de la mort.

Si les décisions qu'a prises Jean-Pierre Lefebvre et que j'ai renversées peuvent dans certains cas se justifier ponctuellement, dans l'isolement du tout, elles ne résistent pas, à mon avis, lorsqu'on cherche à lire et à rendre l'intention du poème, la valeur de chacun des éléments dans le monde du poème dans lequel ils se trouvent et qu'ils constituent tout à la fois. C'est à un tel travail qu'invite une approche herméneutique de la traduction. $\grave{A}$ une interprétation, certes, mais non pas locale, non pas mot à mot, non pas isolée - à une interprétation qui englobe le tout du texte. $\grave{A}$ une écoute de ce que le poème (non l'auteur) dit et fait, à ce qu'il veut dire et être : à son intention.

Interpréter un poème, c'est mettre au jour ce qui a valeur en lui, la dynamique des parties dans le tout. Les éléments ne peuvent être interprétés individuellement, ils ne prennent leur véritable sens que dans la cohabitation avec d'autres et poussent de ce fait dans une direction qui est donnée par chacun des éléments pris dans leur ensemble. Un mot n'a pas dans deux poèmes la même valeur, parce que chaque fois son monde diffère. Ainsi, dans un autre poème traduire ich fürchte mich par J'AI PEUR serait tout à fait indiqué; dans ce poème de Bernhard cette décision manque d'audace, elle demeure en retrait, elle 
reste sourde au poème. L'approche herméneutique de la traduction ne consiste pas à chercher à traduire uniquement le sens, elle cherche à dégager l'intention de son objet et à le recréer. Une bonne traduction sera celle qui aura su faire d'un texte ce qu'il fait dans la sienne. C'est faire un nouveau texte habité par la même intention.

\section{Université de Montréal}

\section{Références}

GADAMER, Hans-Georg (1990). Wahrheit und Methode. Grundzüge einer philosophischen Hermeneutik, tome I des Gesammelte Werke. Tubingue, J.C.B. Mohr (Paul Sibeck). Trad. franç. : Vérité et Méthode. Les grandes lignes d'une herméneutique philosophique. Traduction intégrale revue et complétée par Pierre Fruchon, Jean Grondin et Gilbert Merlio. Paris, Éditions du Seuil, coll. « L'ordre philosophique ».

GREVE, Ludwig (1992). "Sie lacht » und andere Gedichte. Francfortsur-le-Main, Fischer, 77 p.

JACCOTTET, Philippe (1987). Une transaction secrète. Lectures de poésie. Paris, Gallimard. La traduction de Guerne est parue chez Mermod.

KANT, Emmanuel (1985). Critique de la faculté de juger, suivi de Idée d'une histoire universelle au point de vue cosmopolitique et de Réponse à la question: Qu'est-ce que les lumières ? Édition publiée s. la dir. de Ferdinand Alquié, trad. de l'all. par Alexandre J.-.L. Delamarre, JeanRené Ladmiral, Marc B. de Launay, Jean-Marie Vaysse, Luc Ferry et Heinz Wismann. Paris, Gallimard, coll. « Folio/Essais » n 134.

- (1987), Critique de la raison pure. Trad. de l'all. par Jules Barni revue par P. Archambault, préface de Luc Ferry. Paris, GFFlammarion, $\mathrm{n}^{\circ} 257$, XXIII-725 $\mathrm{p}$.

LEFEBVRE, Jean-Pierre (1995) (édition établie par). Anthologie bilingue de la poésie allemande. Préface, chronologie et notes par JeanPierre Lefebvre, Paris, Gallimard, coll. "Bibliothèque de la Pléiade " $\mathrm{n}^{\circ} 401$, (C) 1993 pour les traductions inédites et l'ensemble de l'appareil critique, (C) 1995 pour les traductions inédites et les notes du 
Supplément, pp. 1326-1327. Le poème de Bernhard est tiré du recueil Auf der Erde und in der Hölle, Salzbourg, 1957 (selon la note de la page 1749).

RÉSUMÉ: Herméneutique et traduction poétique: quelques remarques - Une approche herméneutique de la traduction ne doit pas se résumer au simple énoncé selon lequel il faut avoir compris un texte pour le traduire. Elle doit viser à dégager ce que je nomme dans cet article l'intention du texte. Je tente dans ces pages de cerner cette notion d'intention en recourant tout d'abord à une comparaison de la traduction philosophique et poétique, ensuite à l'analyse de deux poèmes : l'un de Ludwig Greve, l'autre de Thomas Bernhard, dont je critique la traduction donnée par Jean-Pierre Lefebvre et duquel je propose une nouvelle version française.

ABSTRACT : Hermeneutics and Poetic Translation: A Few Remarks - An hermeneutical approach to translation should not limit itself to the mere statement that a text needs to be understood in order to be translated. It must rather aim at what I call in this paper the text's intention. In the following pages, I attempt to define this notion of intention through, firstly, a comparison of poetical and philosophical translation, and, secondly, a reading of two poems : one by Laudvig Greve, the other by Thomas Bernhard. In the latter, I criticize JeanPierre Lefebvre's translation and propose my own.

Érick Vigneault : 7074, av. des Érables, Montréal (Québec) H2E 2R2

Courriel : erikv@ctoucomm.qc.ca 